\title{
Fixed Point Theorems in Fuzzy Metric Spaces
}

\author{
Ismat Beg, ${ }^{1}$ Shaban Sedghi, ${ }^{2}$ and Nabi Shobe ${ }^{3}$ \\ ${ }^{1}$ Department of Mathematics and Statistics, Faculty of Management Studies, University of Central Punjab, Lahore, Pakistan \\ ${ }^{2}$ Department of Mathematics, Islamic Azad University, Qaemshahr Branch, Qaemshahr, Iran \\ ${ }^{3}$ Department of Mathematics, Islamic Azad University, Babol Branch, Babol, Iran
}

Correspondence should be addressed to Ismat Beg; ibeg@lums.edu.pk

Received 12 September 2012; Revised 18 October 2012; Accepted 19 October 2012

Academic Editor: Seenith Sivasundaram

Copyright (C) 2013 Ismat Beg et al. This is an open access article distributed under the Creative Commons Attribution License, which permits unrestricted use, distribution, and reproduction in any medium, provided the original work is properly cited.

We prove a fixed point theorem for mappings satisfying an implicit relation in a complete fuzzy metric space.

\section{Introduction and Preliminaries}

The concept of a fuzzy set was introduced by Zadeh [1] in 1965. This concept was used in topology and analysis by many authors. George and Veeramani [2] modified the concept of fuzzy metric space introduced by Kramosil and Michalek [3] and defined the Hausdorff topology of fuzzy metric spaces, which have important applications [4] in quantum particle physics. Afterwards, Beg and Abbas [5], Vasuki [6], Popa [7], and Imad et al. [8, 9] obtained results about fixed point and invariant approximation in fuzzy metric spaces. The aim of this paper is to obtain fixed point of mapping satisfying an implicit relation on fuzzy metric spaces. Our results generalize several known results in the literature.

First, we give some basic definitions.

Definition 1 (see [10]). A binary operation $*:[0,1]^{2} \rightarrow$ $[0,1]$ is called a continuous $t$-norm if $([0,1], *)$ is an abelian topological monoid, that is,

$(1) *$ is associative and commutative,

(2) $*$ is continuous,

(3) $a * 1=a$ for all $a \in[0,1]$,

(4) $a * b \leq c * d$ whenever $a \leq c$ and $b \leq d$, for each $a, b, c, d \in[0,1]$.

Four typical examples of a continuous $t$-norms are $a *{ }_{1} b=$ $\min \{a, b\}, a *_{2} b=a b / \max \{a, b, \lambda\}$ for $0<\lambda<1$ and $a *_{3} b=$ $a b, a *_{4} b=\max \{a+b-1,0\}$.
Definition 2 (see [2]). The 3-tuple $(X, M, *)$ is called a fuzzy metric space if $X$ is an arbitrary nonempty set, * is a continuous $t$-norm, and $M$ is a fuzzy set on $X^{2} \times$ $[0, \infty)$ satisfying the following conditions, for each $x, y, z \in$ $X$ and $t, s>0$,

$$
\begin{aligned}
& (\mathrm{FM}-1) M(x, y, t)>0, \\
& (\mathrm{FM}-2) M(x, y, t)=1 \text { if and only if } x=y, \\
& (\mathrm{FM}-3) M(x, y, t)=M(y, x, t), \\
& (\mathrm{FM}-4) M(x, y, t) * M(y, z, s) \leq M(x, z, t+s), \\
& (\mathrm{FM}-5) M(x, y, \cdot):(0, \infty) \rightarrow[0,1] \text { is continuous. }
\end{aligned}
$$

Let $(X, M, *)$ be a fuzzy metric space. For $t>0$, the open ball $B(x, r, t)$ with a center $x \in X$ and a radius $0<r<1$ is defined by

$$
B(x, r, t)=\{y \in X: M(x, y, t)>1-r\} .
$$

A subset $A \subset X$ is called open if, for each $x \in A$, there exist $t>0$ and $0<r<1$ such that $B(x, r, t) \subset A$. Let $\tau$ denote the family of all open subsets of $X$. Then, $\tau$ is called the topology on $X$ induced by the fuzzy metric $M$. This topology is Hausdorff and first countable. A subset $A$ of $X$ is said to be $F$-bounded if there exist $t>0$ and $0<r<1$ such that $M(x, y, t)>1-r$ for all $x, y \in A$. 
Example 3. Let $X=\mathbb{R}$. Denote $a * b=a b$ for all $a, b \in[0,1]$. For each $t \in(0, \infty)$, define

$$
M(x, y, t)=\frac{t}{t+|x-y|},
$$

for all $x, y \in X$. Then, $(X, M, *)$ is a fuzzy metric space.

Example 4. Let $(X, d)$ be an ordinary metric space and $\psi$ be an increasing and continuous function from $\mathbb{R}_{+}$into $(0,1)$ such that $\lim _{t \rightarrow \infty} \psi(t)=1$. Four typical examples of these functions are $\psi(x)=x /(x+1), \psi(x)=\sin (\pi x /(2 x+1))$, $\psi(x)=1-e^{-x}$, and $\psi(x)=e^{-1 / x}$. Let $a * b \leq a b$ for all $a, b \in[0,1]$. For each $t \in(0, \infty)$, define

$$
M(x, y, t)=[\psi(t)]^{d(x, y)}
$$

for all $x, y \in X$. It is easy to see that $(X, M, *)$ is a fuzzy metric space.

Definition 5 (see [2]). Let $(X, M, *)$ be a fuzzy metric space.

(i) A sequence $\left\{x_{n}\right\}$ in $X$ is said to be convergent to a point $x \in X$ if $\lim _{n \rightarrow \infty} M\left(x_{n}, x, t\right)=1$ for all $t>0$.

(ii) A sequence $\left\{x_{n}\right\}$ in $X$ is called Cauchy sequence if, for each $0<\epsilon<1$ and $t>0$, there exits $n_{0} \in \mathbb{N}$ such that $M\left(x_{n}, x_{m}, t\right)>1-\epsilon$ for each $n, m \geq n_{0}$.

(iii) A fuzzy metric space in which every Cauchy sequence is convergent is said to be complete.

(iv) A fuzzy metric space in which every sequence has a convergent subsequence is said to be compact.

Lemma 6 (see [11]). For all $x, y \in X, M(x, y, \cdot)$ is a nondecreasing function.

Definition 7. Let $(X, M, *)$ be a fuzzy metric space. $M$ is said to be continuous on $X^{2} \times(0, \infty)$ if

$$
\lim _{n \rightarrow \infty} M\left(x_{n}, y_{n}, t_{n}\right)=M(x, y, t),
$$

whenever $\left\{\left(x_{n}, y_{n}, t_{n}\right)\right\}$ is a sequence in $X^{2} \times(0, \infty)$ which converges to a point $(x, y, t) \in X^{2} \times(0, \infty)$, that is,

$$
\begin{gathered}
\lim _{n \rightarrow \infty} M\left(x_{n}, x, t\right)=\lim _{n \rightarrow \infty} M\left(y_{n}, y, t\right)=1, \\
\lim _{n \rightarrow \infty} M\left(x, y, t_{n}\right)=M(x, y, t) .
\end{gathered}
$$

Lemma 8 (see [11]). $M$ is a continuous function on $X^{2} \times$ $(0, \infty)$.

\section{Main Results}

Let $(X, M, *)$ be a fuzzy metric space and $\varnothing \neq S \subseteq X$. Define $\delta_{M}(S, t)=\inf \{M(x, y, t): x, y \in S\}$ for all $t>0$. For an $A_{n}=\left\{x_{n}, x_{n+1}, \ldots\right\}$ in fuzzy metric space $(X, M$, *), let $r_{n}(t)=\delta_{M}\left(A_{n}, t\right)$. Then, $r_{n}(t)$ is finite for all $n \in \mathbf{N},\left\{r_{n}(t)\right\}$ is nonincreasing, $r_{n}(t) \rightarrow r(t)$ for some $0 \leq r(t) \leq 1$, and also $r_{n}(t) \leq M\left(x_{l}, x_{k}, t\right)$ for all $l, k \geq n$.
Let $\mathscr{F}$ be the set of all continuous functions $F:[0,1]^{3} \times$ $[0,1] \rightarrow[-1,1]$ such that $F$ is nondecreasing on $[0,1]^{3}$ satisfying the following conditions.

$\left(F_{1}\right): F((u, u, u), v) \leq 0$ implies that $v \geq \gamma(u)$ where $\gamma:[0,1] \rightarrow[0,1]$ is a nondecreasing continuous function with $\gamma(s)>s$ for $s \in[0,1)$.

Example 9. $F:[0,1]^{3} \times[0,1] \rightarrow[-1,1]$, defined the following:

(i) $F\left(\left(t_{1}, t_{2}, t_{3}\right), t_{4}\right)=\gamma\left(\min \left\{t_{1}, t_{2}, t_{3}\right\}\right)-t_{4}$,

(ii) $F\left(\left(t_{1}, t_{2}, t_{3}\right), t_{4}\right)=\gamma\left(\sum_{i=1}^{3} a_{i} t_{i}\right)-t_{4}$ such that for every $a_{i} \geq 0, \sum_{i=1}^{3} a_{i}=1$, where $\gamma(s)=s^{h}$ for $0<h<1$.

In this paper, our main result is the following theorem.

Theorem 10. Let $(X, M, *)$ be a complete bounded fuzzy metric space and $T$ a self map of $X$ satisfying for all $x, y \in X$ the implicit relation

(i)

$$
\begin{aligned}
& F(M(x, y, t), M(T x, x, t), M(T x, y, t), M(T x, T y, t)) \\
& \quad \leq 0
\end{aligned}
$$

where $F \in \mathscr{F}$.

Then, $T$ has a unique fixed point $p$ in $X$, and $T$ is continuous at $p$.

Proof. Let $x_{0} \in X$ and $T x_{n}=x_{n+1}$. Let $r_{n}(t)=\delta_{M}\left(A_{n}, t\right)$, where $A_{n}=\left\{x_{n}, x_{n+1}, \ldots\right\}$. Then, we know $\lim _{n \rightarrow \infty} r_{n}(t)=$ $r(t)$ for some $0 \leq r(t) \leq 1$. If $x_{n+1}=x_{n}$ for some $n \in \mathbf{N}$, then $T$ has a fixed point, $p \in X$. Assume that $x_{n+1} \neq x_{n}$ for each $n \in \mathbf{N}$. Let $k \in \mathbf{N}$ be fixed. Taking $x=x_{n-1}, y=x_{n+m-1}$ in (i) where $n \geq k$ and $m \in \mathbf{N}$, we have

$$
\begin{gathered}
F\left(M\left(x_{n-1}, x_{n+m-1}, t\right), M\left(T x_{n-1}, x_{n-1}, t\right),\right. \\
\left.M\left(T x_{n-1}, x_{n+m-1}, t\right), M\left(T x_{n-1}, T x_{n+m-1}, t\right)\right) \\
=F\left(M\left(x_{n-1}, x_{n+m-1}, t\right), M\left(x_{n}, x_{n-1}, t\right),\right. \\
\left.M\left(x_{n}, x_{n+m-1}, t\right), M\left(x_{n}, x_{n+m}, t\right)\right) \leq 0 .
\end{gathered}
$$

Thus, we have

$$
F\left(r_{n-1}(t), r_{n-1}(t), r_{n}(t), M\left(x_{n}, x_{n+m}, t\right)\right) \leq 0,
$$

since $F$ is nondecreasing on $[0,1]^{3}$. Also, since $r_{n}(t)$ is nonincreasing, we have

$$
F\left(r_{k-1}(t), r_{k-1}(t), r_{k-1}(t), M\left(x_{n}, x_{n+m}, t\right)\right) \leq 0,
$$

which implies that

$$
M\left(x_{n}, x_{n+m}, t\right) \geq \gamma\left(r_{n-1}(t)\right) .
$$

Thus, for all $n \geq k$, we have $\inf _{n \geq k}\left\{M\left(x_{n}, x_{n+m}, t\right)\right\}=r_{k}(t) \geq$ $\gamma\left(r_{n-1}(t)\right)$. Letting $k \rightarrow \infty$, we get $r(t) \geq \gamma(r(t))$. If $r(t) \neq 1$, then $r(t) \geq \gamma(r(t))>r(t)$, which is a contradiction. Thus 
$r(t)=1$ and hence $\lim _{n \rightarrow \infty} \gamma_{n}(t)=1$. Thus, given $\epsilon>0$, there exists an $n_{0} \in \mathbf{N}$ such that $r_{n}(t)>1-\epsilon$. Then, we have for $n \geq n_{0}$ and $m \in \mathbf{N}, M\left(x_{n}, x_{n+m}, t\right)>1-\epsilon$. Therefore, $\left\{x_{n}\right\}$ is a Cauchy sequence in $X$. By the completeness of $X$, there exists a $p \in X$ such that $\lim _{n \rightarrow \infty} T x_{n}=\lim _{n \rightarrow \infty} x_{n+1}=p$. Taking $x=x_{n}, y=p$ in (i), we have

$$
\begin{aligned}
F( & M\left(x_{n}, p, t\right), M\left(T x_{n}, p, t\right), \\
& \left.M\left(T x_{n}, x_{n}, t\right), M\left(T x_{n}, T p, t\right)\right) \\
= & F\left(M\left(x_{n}, p, t\right), M\left(x_{n+1}, p, t\right),\right. \\
& \left.M\left(x_{n+1}, x_{n}, t\right), M\left(x_{n+1}, T p, t\right)\right) \leq 0 .
\end{aligned}
$$

Taking $\lim _{n \rightarrow \infty}$, we have

$$
F(M(p, p, t), M(p, p, t), M(p, p, t), M(p, T p, t)) \leq 0,
$$

which implies that $M(p, T p, t) \geq \gamma(M(p, p, t))=\gamma(1)=1$. Hence, $T p=p$. For the uniqueness, let $p$ and $w$ be fixed points of $T$. Taking $x=p, y=w$ in (i), we have

$$
\begin{aligned}
F & (M(p, w, t), M(T p, p, t), M(T p, w, t), M(T p, T w, t)) \\
& =F(M(p, w, t), M(p, p, t), M(p, w, t), M(p, w, t)) \\
& \leq 0 .
\end{aligned}
$$

Since $F$ is nondecreasing on $[0,1]^{3}$, we have

$$
F(M(p, w, t), M(p, w, t), M(p, w, t), M(p, w, t)) \leq 0,
$$

which implies that $M(p, w, t) \geq \gamma(M(p, w, t))>M(p, w, t)$, which is a contradiction. Thus, we have $p=w$. Now, we show that $T$ is continuous at $p$. Let $\left\{y_{n}\right\}$ be a sequence in $X$ and $\lim _{n \rightarrow \infty} y_{n}=p$. Taking $x=p, y=y_{n}$ in (i), we have

$$
\begin{aligned}
F & \left(M\left(p, y_{n}, t\right), M(T p, p, t), M\left(T p, y_{n}, t\right), M\left(T p, T y_{n}, t\right)\right) \\
& =F\left(M\left(p, y_{n}, t\right), M(p, p, t), M\left(p, y_{n}, t\right), M\left(p, T y_{n}, t\right)\right) \\
& \leq 0,
\end{aligned}
$$

which implies that $\gamma\left(M\left(p, T y_{n}, t\right)\right) \geq \gamma\left(M\left(p, y_{n}, t\right)\right)$. Taking limit inf, we have

$$
\begin{aligned}
\liminf _{n}\left\{\gamma\left(M\left(p, T y_{n}, t\right)\right)\right\} & \geq \liminf _{n} \gamma\left(M\left(p, y_{n}, t\right)\right) \\
& =\gamma(1)=1 .
\end{aligned}
$$

Thus, $\lim _{n \rightarrow \infty} T y_{n}=p=T p$. Hence, $T$ is continuous at p.

Corollary 11. Let $(X, M, *)$ be a complete bounded fuzzy metric space, $m \in \mathbf{N}$, and $T$ a self map of $X$ satisfying for all $x, y \in X$, (i)

$$
\begin{gathered}
F\left(M(x, y, t), M\left(T^{m} x, x, t\right), M\left(T^{m} x, y, t\right),\right. \\
\left.M\left(T^{m} x, T^{m} y, t\right)\right) \leq 0,
\end{gathered}
$$

where $F \in \mathscr{F}$.

Then, $T$ has a unique fixed point $p$ in $X$, and $T^{m}$ is continuous at $p$.

Proof. From Theorem 10, $T^{m}$ has a unique fixed point $p$ in $X$ and $T^{m}$ is continuous at $p$. Since $T p=T T^{m} p=T^{m} T p$, $T p$ is also a fixed point of $T^{m}$. By the uniqueness, it follows $T p=p$.

In Theorem 10 (Corollary 11, resp.), If we take $F\left(\left(t_{1}, t_{2}, t_{3}\right), t_{4}\right)=\gamma\left(\min \left\{t_{1}, t_{2}, t_{3}\right\}\right)-t_{4}$, then we have the next result.

Corollary 12. Let $(X, M, *)$ be a complete bounded fuzzy metric space and $T$ a self map of $X$ satisfying for all $x, y \in X$,

(i)

$$
\begin{aligned}
& M(T x, T y, t) \\
& \quad \geq \gamma(\min \{M(x, y, t), M(T x, x, t), M(T x, y, t)\}) .
\end{aligned}
$$

Then, $T$ has a unique fixed point $p$ in $X$, and $T$ is continuous at $p$.

Example 13. Let $X=\mathbf{R}$. Define $M: X^{2} \times[0, \infty) \rightarrow \mathbf{R}^{+}$ by $M(x, y, t)=e^{-|x-y| / t}$ for all $x, y \in X$ and $t>0$. Then, $(X, M, *)$ is a complete fuzzy metric space where $a * b=a b$. Define map $T: X \rightarrow X$ by $T(x)=x / 2$ for $x \in X$ and let $\gamma:[0,1] \rightarrow[0,1]$ defined by $\gamma(s)=s^{1 / 2}$. It is easy to see that

$$
\begin{aligned}
M & (T x, T y, t)=e^{-|x / 2-y / 2| / t} \\
& =\sqrt{e^{-|x-y| / t}}=\sqrt{M(x, y, t)} \\
& \geq \sqrt{\min \{M(x, y, t), M(T x, x, t), M(T x, y, t)} .
\end{aligned}
$$

Thus, $T$ satisfy all the hypotheses of Corollary 12 , and hence $T$ has a unique fixed point. Indeed, 0 is the unique fixed point of $T$.

Corollary 14. Let $(X, M, *)$ be a complete bounded fuzzy metric space, $m \in \mathbf{N}$, and $T$ a self map of $X$ satisfying for all $x, y \in X$

(i)

$$
\begin{aligned}
M & \left(T^{m} x, T^{m} y, t\right) \\
& \geq \gamma\left(\min \left\{M(x, y, t), M\left(T^{m} x, x, t\right), M\left(T^{m} x, y, t\right)\right\}\right) .
\end{aligned}
$$

Then, $T$ has a unique fixed point $p$ in $X$, and $T^{m}$ is continuous at $p$. 
Corollary 15. Let $(X, M, *)$ be a complete bounded fuzzy metric space and $T$ a self map of $X$ satisfying for all $x, y \in X$,

(i)

$$
\begin{aligned}
& M(T x, T y, t) \\
& \quad \geq \sqrt{a_{1} M(x, y, t)+a_{2} M(T x, x, t)+a_{3} M(T x, y, t)},
\end{aligned}
$$

such that for every $a_{i} \geq 0, \sum_{i=1}^{3} a_{i}=1$. Then, $T$ has a unique fixed point $p$ in $X$, and $T$ is continuous at $p$.

Let $\mathscr{F}$ be the class of all continuous functions $F:[0,1]^{3} \times$ $[0,1] \rightarrow[-1,1]$ such that $F$ is nondecreasing on $[0,1]^{3}$ satisfying $F((u, u, u), v)<0$ implies that $v>u$.

Example 16. Let $F:[0,1]^{3} \times[0,1] \rightarrow[-1,1]$ defined by $F\left(\left(t_{1}, t_{2}, t_{3}\right), t_{4}\right)=\left(\min \left\{t_{1}, t_{2}, t_{3}\right\}\right)^{h}-t_{4}$ for some $0<h<1$. Then, $F$ is continuous on $[0,1]^{3}$ and nondecreasing on $[0,1]^{3}$. Also, we have that $F((u, u, u), v)<0$ implies that $v>u$. Thus $F \in \mathscr{F}$.

Theorem 17. Let $(X, M, *)$ be a compact fuzzy metric space and $T$ a continuous self map of $X$ satisfying for all $x, y \in X$ with

$$
\begin{aligned}
& F(M(x, y, t), M(T x, x, t), M(T x, y, t), M(T x, T y, t)) \\
& \quad<0,
\end{aligned}
$$

where $F \in \mathscr{F}$.

Then, $T$ has a unique fixed point $p$ in $X$.

Proof. We know that for $n=1,2, \ldots, T^{n} X$ is compact and $T^{n+1} X \subset T^{n} X$. Let $X_{0}=\bigcap_{n=1}^{\infty} T^{n} X$. Then, $X_{0}$ is a nonempty compact subset of $X$, and $T X_{0}=X_{0}$. We claim that $X_{0}$ is a singleton set. Suppose $X_{0}$ is not singleton. Then, we know that the function $M: X^{2} \times(0, \infty) \rightarrow[-1,1]$ has a minimum value. That is, there exists a $\left(x_{0}, y_{0}, t\right) \in X_{0}^{2} \times(0, \infty)$ such that $M\left(x_{0}, y_{0}, t\right) \leq M(x, y, t)$ for all $x, y \in X_{0}$. Since $T X_{0}=X_{0}$, there exist $x_{1}, y_{1} \in X_{0}$ such that $T x_{1}=x_{0}, T y_{1}=y_{0}$. Thus, we have

$$
\begin{aligned}
& F\left(M\left(x_{1}, y_{1}, t\right), M\left(x_{0}, x_{1}, t\right), M\left(x_{0}, y_{1}, t\right), M\left(x_{0}, y_{0}, t\right)\right) \\
& \quad<0,
\end{aligned}
$$

and so $F\left(M\left(x_{0}, y_{0}, t\right), M\left(x_{0}, y_{0}, t\right), M\left(x_{0}, y_{0}, t\right), M\left(x_{0}, y_{0}, t\right)\right)$ $<0$ which implies that $M\left(x_{0}, y_{0}, t\right)>M\left(x_{0}, y_{0}, t\right)$. This is a contradiction. Thus, $X_{0}$ is singleton, and hence $T$ has a fixed point in $X$. Uniqueness of fixed point of $T$ follows from (i).

Corollary 18. Let $(X, M, *)$ be a compact fuzzy metric space, $m \in \mathbf{N}$, and $T^{m}$ a continuous self map of $X$ satisfying for all $x, y \in X$, (i)

$$
\begin{gathered}
F\left(M(x, y, t), M\left(T^{m} x, x, t\right), M\left(T^{m} x, y, t\right),\right. \\
\left.M\left(T^{m} x, T^{m} y, t\right)\right)<0,
\end{gathered}
$$

where $F \in \mathscr{F}$.

Then, $T$ has a unique fixed point $p$ in $X$.

Proof. From Theorem 17, $T^{m}$ has a unique fixed point $p$ in $X$. Since $T p=T T^{m} p=T^{m} T p, T p$ is also a fixed point of $T^{m}$. By the uniqueness, it follows $T p=p$.

\section{References}

[1] L. A. Zadeh, "Fuzzy sets," Information and Control, vol. 8, no. 3, pp. 338-353, 1965.

[2] A. George and P. Veeramani, "On some results in fuzzy metric spaces," Fuzzy Sets and Systems, vol. 64, no. 3, pp. 395-399, 1994.

[3] I. Kramosil and J. Michalek, "Fuzzy metric and statistical metric spaces," Kybernetika, vol. 11, no. 5, pp. 336-344, 1975.

[4] Y. Tanaka, Y. Mizuno, and T. Kado, "Chaotic dynamics in the Friedmann equation," Chaos, Solitons and Fractals, vol. 24, no. 2, pp. 407-422, 2005.

[5] I. Beg and M. Abbas, "Invariant approximation for fuzzy nonexpansive mappings," Mathematica Bohemica, vol. 136, no. 1, pp. 51-59, 2011.

[6] R. Vasuki, "Common fixed points for R-weakly commuting maps in fuzzy metric spaces," Indian Journal of Pure and Applied Mathematics, vol. 30, no. 4, pp. 419-423, 1999.

[7] V. Popa, "Some fixed point theorems for compatible mappings satisfying an implicit relation," Demonstratio Mathematica, vol. 32, no. 1, pp. 157-163, 1999.

[8] M. Imdad and J. Ali, "A general fixed point theorem in fuzzy metric spaces via an implicit function," Journal of Applied Mathematics \& Informatics, vol. 26, no. 3-4, pp. 591-603, 2008.

[9] M. Imdad, Kumar, Santosh, and M. S. Khan, "Remarks on some fixed point theorems satisfying implicit relations. Dedicated to the memory of Prof. Dr. Naza Tanovic-Miller," Radovi Matematički, vol. 11, no. 1, pp. 135-143, 2002.

[10] B. Schweizer and A. Sklar, "Statistical metric spaces," Pacific Journal of Mathematics, vol. 10, pp. 313-334, 1960.

[11] M. Grabiec, "Fixed points in fuzzy metric spaces," Fuzzy Sets and Systems, vol. 27, no. 3, pp. 385-389, 1988. 


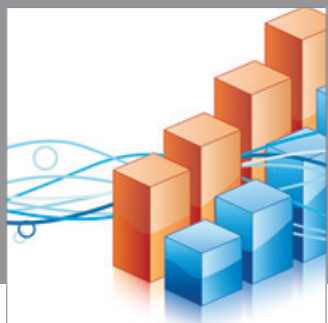

Advances in

Operations Research

mansans

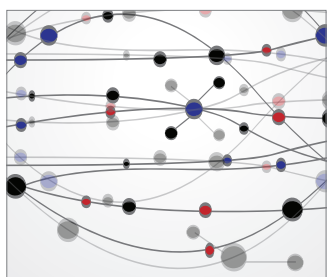

The Scientific World Journal
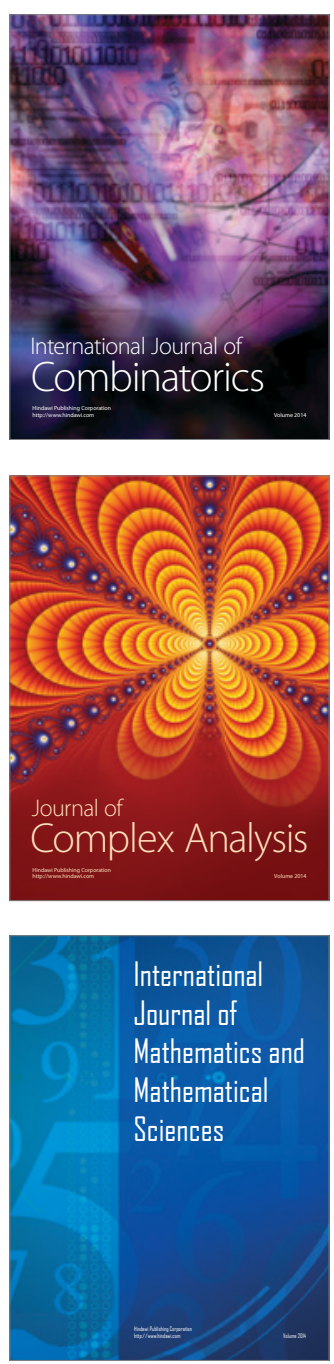
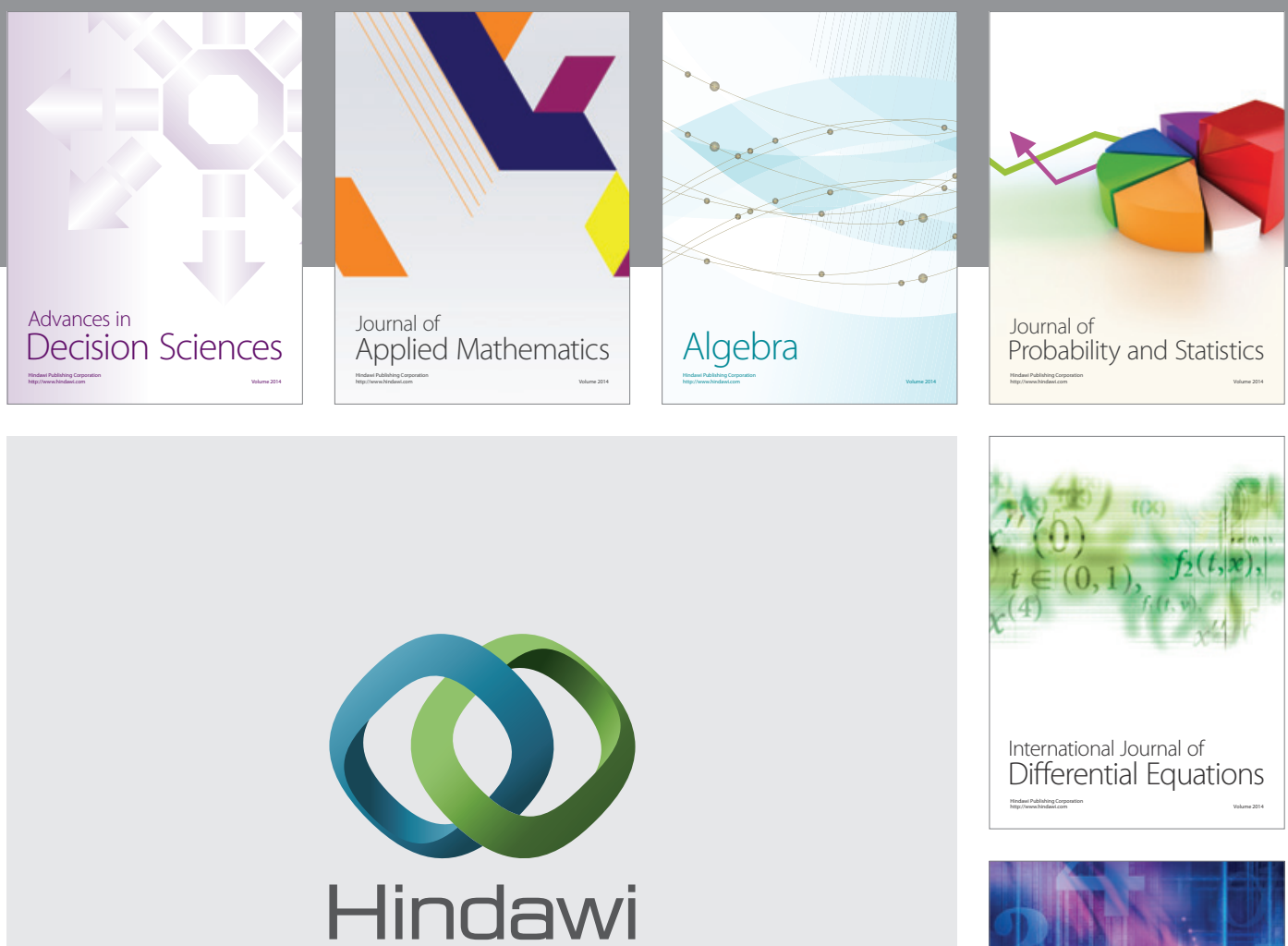

Submit your manuscripts at http://www.hindawi.com
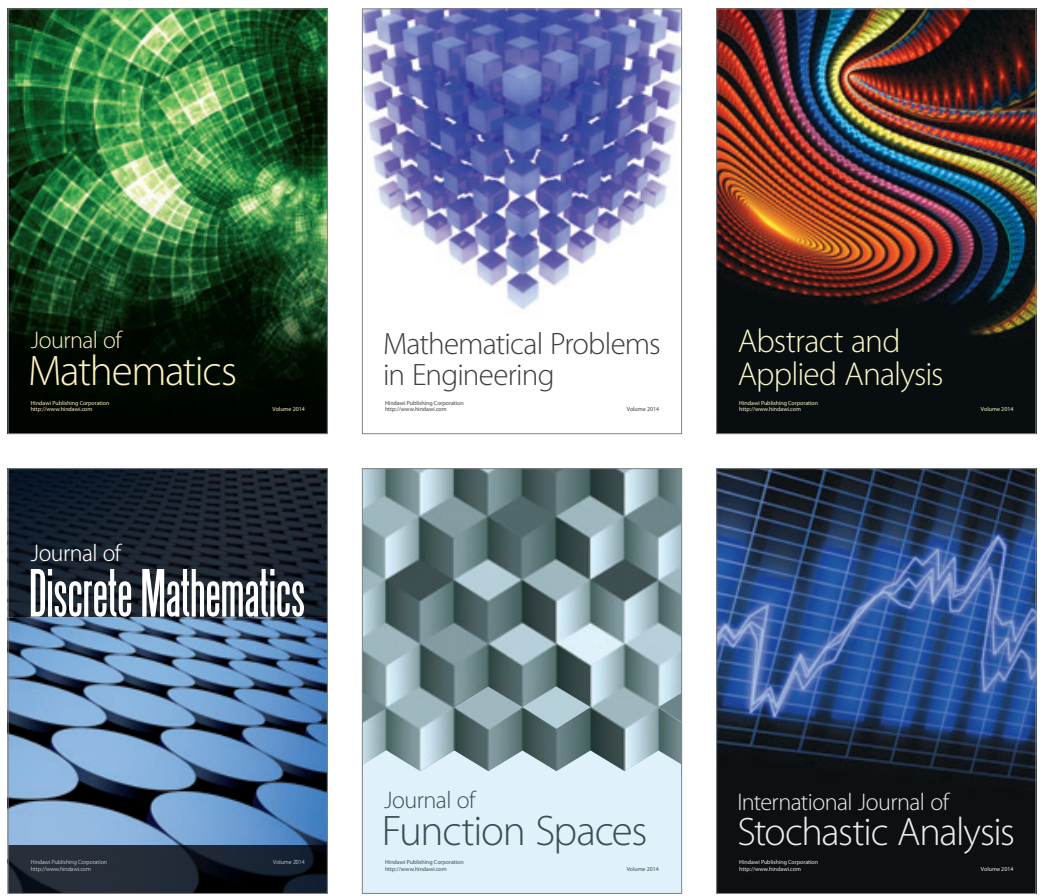

Journal of

Function Spaces

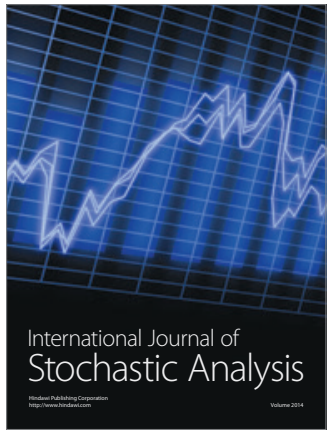

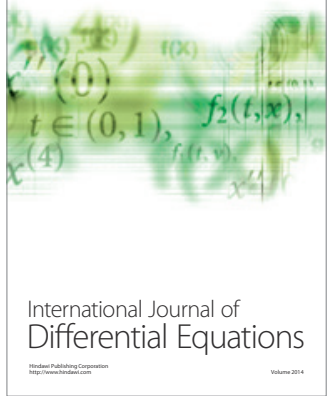
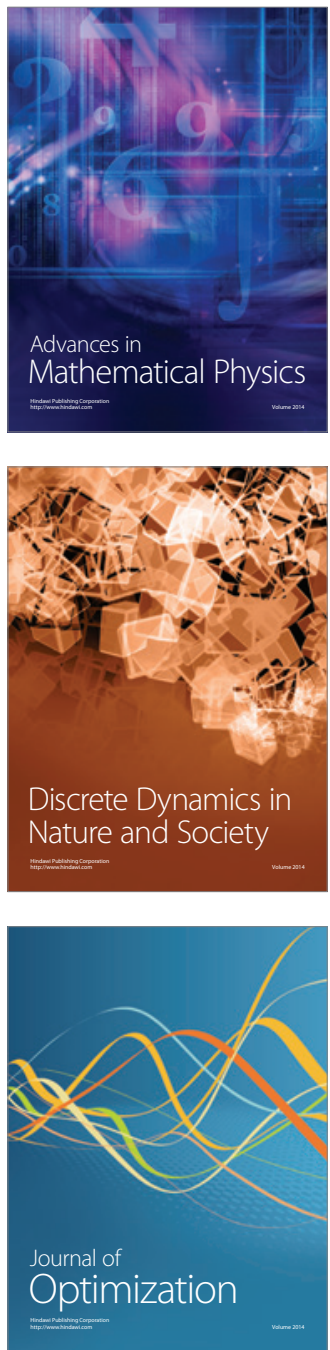